\title{
Influence of Internal Heat Generation/Absorption, Thermal Radiation, Magnetic Field, Variable Fluid Property and Viscous Dissipation on Heat Transfer Characteristics of a Maxwell Fluid over a Stretching Sheet
}

\author{
K. V. Prasad ${ }^{1 \dagger}$, K. Vajravelu ${ }^{2}$ and A. Sujatha ${ }^{1}$ \\ ${ }^{1}$ Department of Mathematics, VSK University, Vinayaka Nagar, Bellary- 583 104, Karnataka, India \\ ${ }^{2}$ Department of Mathematics, University of Central Florida, Orlando, Florida 32816, USA
}

$\uparrow$ Corresponding Author Email:

(Received August 3, 2011; accepted October 9, 2011)

\begin{abstract}
The present study investigates the effects of internal heat generation/absorption, thermal radiation, magnetic field, and temperature-dependent thermal conductivity on the flow and heat transfer characteristics of a Non-Newtonian Maxwell fluid over a stretching sheet. The upper convected Maxwell fluid model is used to characterize the nonNewtonian fluid behavior. Similarity solutions for the governing equations are obtained with prescribed surface temperature (PST) and/or with prescribed surface heat flux (PHF). Numerical solutions for the governing equations subject to the appropriate boundary conditions are obtained by a finite difference scheme known as Keller-Box method. The numerical results thus obtained are analyzed for the effects of the several pertinent parameters namely, the Maxwell parameter, the magnetic parameter, the temperature-dependent thermal conductivity parameter, the heat source/sink parameter, the Prandtl number, the Eckert number, and the thermal radiation parameter on the flow and heat transfer fields. Results for the velocity and temperature fields, skin friction, and Nusselt number are shown through graphs. It is observed that the thermal boundary layer thickness increases with increasing values of the elasticity parameter and the magnetic parameter; however it decreases with the Prandtl number.
\end{abstract}

Keywords: Maxwell fluid, Magneto-hydro-dynamic flow, Variable thermal conductivity, Thermal radiation, Viscous dissipation, Keller-Box method.

\section{INTRODUCTION}

During the past three decades, the flow of an incompressible viscous fluid over a stretching sheet has acquired special attention because of its many industrial applications (see for details, Agassant et al. 1991 and Bird et al. 1987). In particular, flow of this kind occurs in a cooling bath, the boundary layer along a material handling conveyers, the aerodynamic extrusion of plastic sheets, the boundary layer along liquid film and condensation processes, the cooling or drying of papers and textiles, and glass fiber production. This type of flow investigation was initiated by Sakiadis (1961) and extended by Crane (1970) to fluid flow over a linearly stretched sheet. Later works on the stretching sheet problems to Newtonian fluid models by taking into account different physical situations are extensively analyzed by several authors (see Gupta and Gupta 1977, Grubka and Bobba 1985, Cortell 2005, Vleggaar 1986, Chen and Char 1988, Liu 2005). However, many industrial fluids are non-Newtonian such as molten plastics, polymers, suspension, foods, slurries, paints, glues, printing inks, blood. That is, they may exhibit dynamic deviation from Newtonian behavior depending upon the flow configuration and/or the rate of deformation. These fluids often obey non-linear constitutive equations and the complexity of their constitutive equation is the main culprit for the lack of exact analytical solution. For example, visco-elastic fluid models used in these works are simple models; whereas, second order fluid model and Walters' model (Rajagopal et al. 1984, Siddappa and Subhas Abel 1985, Cortell 2006, Andersson 1992, Subhas Abel et al. 2008) which are known to be good for weakly elastic fluids subjected to slowly varying flows. These two models are known to violate certain rules of thermodynamics, and virtually all of them are based on the boundary layer theory which is still incomplete for non-Newtonian fluids. Therefore significance of the results reported in the above works is limited as far as the polymer industry is concerned. Obviously for the theoretical results to be of any industrial importance, more general visco-elastic fluid models such as upper convected Maxwell (UCM) model or Oldroyd B model should be invoked in the analysis. Indeed, these two fluid models have been recently used to study the flow 
of visco-elastic fluids above stretching and nonstretching sheets with or without heat transfer (Bhatnagar et al. 1995, Renardy 1997, Sadeghy et al. 2005, Hayat et al. 2006, Aliakbar et al. 2009).

In all the above mentioned studies, the thermo-physical properties of the ambient fluids were assumed to be constant. However it is well known that these properties may change with temperature, especially the thermal conductivity. Available literature (Chaim 1998, Govindarajulu and Thangaraj 1987, Subhas Abel and Mahesha 2008, Savvas et al. 1994) on variable thermal conductivity shows that this type of work has not been carried out for non-Newtonian UCM fluid in the presence a transverse magnetic field. This type of flow finds applications in polymer industry (where one deals with stretching of plastic sheets) and metallurgy where hydro-magnetic techniques are being used. To be more specific, it may be pointed out that many metallurgical processes involve the cooling of continuous strips or filaments by drawing them through a quiescent fluid and that in the process of drawing, these strips are sometimes stretched. Mention may be made of drawing, annealing, and thinning of copper wires. In all these cases, the properties of final product depend to a great extent on the rate of cooling by drawing such strips in an electrically conducting fluid subject to a uniform magnetic field. Another important application of hydromagnetic flow to metallurgy lies in the purification of molten metals from non-metallic inclusion.

Therefore, in the present paper we study the effects of variable thermal conductivity, and thermal radiation on the heat transfer of a non-Newtonian UCM fluid over a non-isothermal stretching sheet in the presence of internal heat generation/absorption and viscous dissipation, subjected to a transverse magnetic field. Savvas et al. (1994) suggested that for liquid metals, the thermal conductivity varies linearly with temperature in the range of $0^{\circ} \mathrm{F}$ to $400^{\circ} \mathrm{F}$. Hence, we assume that the thermal conductivity is a linear function of the temperature. Because of the rheological equation of state, the momentum and energy equations are highly non-linear partial differential equations (PDEs). These PDEs are converted to non-linear ordinary differential equations (ODEs) by using a similarity transformation. Because of the complexity and the non-linearly in the proposed problem, it is solved numerically by the Keller-Box method. Numerical computation is carried out for temperature and horizontal velocity fields, the Nusselt number and the skin friction for two general cases of non-isothermal boundary conditions. The effects of the different physical parameters on the flow phenomenon and the heat transfer process are presented through graphs and the results are discussed.

\section{NUMERICAL FORMULATION}

We consider a steady, laminar, two-dimensional flow of an incompressible, electrically conducting nonNewtonian upper convected Maxwell fluid (in the presence of a transverse magnetic field) over a nonisothermal stretching sheet. The flow is generated due to the stretching of an elastic sheet caused by the simultaneous application of two equal and opposite forces along the $x$-axis, keeping the origin fixed and considering the flow to be confined to the region $y>0$. The thermo-physical properties of the sheet and the ambient fluid are assumed to be constant. The flow is subject to a uniform magnetic field of strength $B_{0}$ applied normal to the surface. It is assumed that the sheet moves with linear velocity $u=b x$, where $b$ is the linear stretching rate, $x$ is the distance from the sheet. It is also assumed that the magnetic Reynolds number is very small, further since there is no electric field; the electric field due to polarization of charges is negligible. It is assumed that boundary layer approximation is applicable in our case Gupta and Wineman (1980). Therefore, the first step would be to derive the boundary layer equations for our fluid of interest in this particular geometry, and this can be done starting from Cauchy equations of motion in which a source term due to the magnetic field should also be included Bird et al. (1987). For a two-dimensional flow, the equation of continuity and the equations of motion with no pressure gradient present can be written as:

$\frac{\partial u}{\partial x}+\frac{\partial \mathrm{v}}{\partial y}=0$

$\rho\left(u \frac{\partial u}{\partial x}+\mathrm{v} \frac{\partial u}{\partial y}\right)=\frac{\partial \tau_{x x}}{\partial x}+\frac{\partial \tau_{x y}}{\partial y}-\sigma B_{0}^{2} u$

$\rho\left(u \frac{\partial \mathrm{v}}{\partial x}+\mathrm{v} \frac{\partial \mathrm{v}}{\partial y}\right)=\frac{\partial \tau_{x x}}{\partial x}+\frac{\partial \tau_{y y}}{\partial y}$

where $u$ and $\mathrm{v}$ are the velocities components along the $x$ and $y$ axes respectively, $\rho$ is the fluid density, $\sigma$ is the electrical conductivity, and $B_{0}$ is the uniform magnetic field. As mentioned above, the fluid of interest in the present work obeys upper convected Maxwell model. For a Maxwell fluid the extra tensor $\tau_{i j}$ can be related to the deformation rate tensor $d_{i j}$ by an equation of the form

$\tau_{i j}+\lambda \frac{\Delta}{\Delta t} \tau_{i j}=2 \delta d_{i j}$

where $\delta$ is the coefficient of viscosity and $\lambda$ is the relaxation time of the period. The time derivative $\frac{\Delta}{\Delta t}$ appearing in the above equation is the so called upper convected time derivative devised to satisfy the requirements of the continuum mechanics (i.e., material objectivity and frame difference). This time derivative when applied to stress tensor reads as follows (Bird et al. 1987).

$\frac{\Delta}{\Delta t} \tau_{i j}=\frac{D}{D t} \tau_{i j}-L_{j k} \tau_{i k}-L_{i k} \tau_{k j}$

where $L_{i j}$ is the velocity gradient tensor. For an incompressible fluid obeying Upper convected Maxwell model, the $x$-momentum equation can be simplified using the boundary layer theory as (Sadeghy et al. 2005):

$u \frac{\partial u}{\partial x}+\mathrm{v} \frac{\partial u}{\partial y}+\lambda\left(u^{2} \frac{\partial^{2} u}{\partial x^{2}}+\mathrm{v}^{2} \frac{\partial^{2} \mathrm{v}}{\partial y^{2}}+2 u \mathrm{v} \frac{\partial^{2} u}{\partial x \partial y}\right)=\gamma \frac{\partial^{2} u}{\partial y^{2}}-\frac{\sigma B_{0}^{2} u}{\rho}$, 
where $\gamma$ is the kinematic viscosity of the fluid, The appropriate boundary conditions for the problem are:

$u(x, 0)=b x, \mathrm{v}(x, 0)=0$,

$u(x, y) \rightarrow 0$ as $y \rightarrow \infty$.

To solve the above boundary layer equations the following similarity transformation are introduced:

$u=b x f^{\prime}(\eta), \quad \mathrm{v}=-\sqrt{b \gamma} \quad f(\eta), \quad \eta=\sqrt{\frac{b}{\gamma}} \quad y$.

Here, $f(\eta)$ is the dimensionless stream function and $\eta$ is the similarity variable. The velocity components $u$ and $\mathrm{v}$ in Eq. (8) automatically satisfies the continuity Eq. (1). In terms of $f$, the momentum Eq. (6) can be written as

$f^{\prime \prime \prime}-M n f^{\prime}-f^{\prime 2}+f f^{\prime \prime}+\beta\left\{2 f f^{\prime} f^{\prime \prime}-f^{2} f^{\prime \prime \prime}\right\}=0$,

where, $M n=\frac{\sigma B_{0}^{2}}{\rho b}$ is the magnetic parameter and

$\beta=\lambda b$ is the Maxwell parameter. In view of the transformations, the boundary conditions (7) can be written as

$$
\begin{aligned}
& f(\eta)=0, f^{\prime}(\eta)=1 \quad \text { at } \eta=0, \\
& \lim _{\eta \rightarrow \infty} f^{\prime}(\eta) \rightarrow 0 .
\end{aligned}
$$

It is worth mentioning here that for the Sakiadis flow of a second grade fluid, we would get a fourth order differential equation with only three boundary conditions. For the second grade fluid flow case, augmenting the needed boundary conditions to match the order of the differential equation has turned out to be a big issue (for details see Rajagopal and Gupta 1980, Garg and Rajagopal 1991). Fortunately, here in spite of the fact that the Maxwell model is much more involved than the second-grade model, Sakiadis flow provides a much simpler fluid mechanical problem to be solved. The exact solution of Eq. (9) with the boundary conditions (10) for $\beta=0$ is obtained as

$$
f(\eta)=\frac{1-e^{-\alpha \eta}}{\alpha}, \alpha>0
$$

where $\alpha=\sqrt{1+M n}$

The shear stress at the sheet is

$$
\tau_{0}=-\mu\left(\frac{\partial u}{\partial y}\right)_{y=0},
$$

and its dimensionless form is

$$
\tau=\frac{\tau_{0}}{b^{2} x^{2} \rho} \text {. }
$$

\section{Heat Transfer AnAlysis}

The energy equation with variable thermal conductivity in the presence of internal heat generation/absorption, viscous dissipation and thermal radiation for twodimensional boundary layer UCM fluid flow (Chaim 1998, Brewster 1992, Raptis and Perdikis 1991, and Raptis 1999) is given by: $u \frac{\partial T}{\partial x}+\mathrm{v} \frac{\partial T}{\partial y}=\frac{1}{\rho c_{p}} \frac{\partial}{\partial y}\left(k(T) \frac{\partial T}{\partial y}\right)+$

$\frac{Q_{s}}{\rho c_{p}}\left(T-T_{\infty}\right)+\frac{\mu}{\rho c_{p}}\left(\frac{\partial u}{\partial y}\right)^{2}-\frac{1}{\rho c_{p}} \frac{\partial q_{r}}{\partial y}$,

where $T$ is the temperature, $C_{p}$ is the specific heat at constant pressure, $k$ is the thermal conductivity. In this paper thermal conductivity is assumed to vary as a linear function of temperature (Chaim 1998) as

$k(T)=k_{\infty}\left(1+\frac{\varepsilon}{\Delta T}\left(T-T_{\infty}\right)\right)$.

In Eq. (15), $\Delta T=T_{w}-T_{\infty}, T_{w}$ is the sheet temperature, $\varepsilon$ is a small parameter and $\kappa_{\infty}$ is the conductivity of the fluid far away from the sheet. The second term containing $Q_{s}$ in the right hand side (RHS) of Eq. (14) represents the temperature dependent volumetric rate of heat source when $Q_{s}>0$ and heat sink when $Q_{s}<0$. These heat sources and sinks deal with the situations of exothermic and endothermic chemical reactions respectively. Viscous dissipation or frictional heating term is accounted in the heat transfer analysis by $\frac{\mu}{\rho c_{p}}\left(\frac{\partial u}{\partial y}\right)^{2}$ with the assumption that UCM fluid is more viscous in nature than elastic: Due to this assumption we neglect elastic deformation in comparison with the viscous dissipation. The last term $q_{r}$ in the RHS of Eq. (14) is the radiative heat flux and is given by

$q_{r}=\frac{-4 \sigma^{*}}{3 K^{*}} \frac{\partial T^{4}}{\partial y}$,

where $\sigma^{*}$ and $K^{*}$ are respectively the StephanBoltzmann constant and the mean absorption coefficient. We assume that the temperature field within the fluid is of the form $T^{4}$ and may be expanded in Taylor series about $T_{\infty}$. Neglecting the higher order terms, we obtain $T^{4} \cong 4 T_{\infty}^{3}-3 T_{\infty}^{4}$ and using this expression for $T^{4}$ in Eq. (16) we get

$q_{r}=\frac{-16 \sigma^{*} T_{\infty}^{3}}{3 K^{*}} \frac{\partial T}{\partial y}$.

Substituting Eqs. (15), (16) and (17) into Eq. (14) we get

$$
\begin{gathered}
\rho c{ }_{p} u \frac{\partial T}{\partial x}+\left(\rho c_{p} \mathrm{v}-\frac{k_{\infty} \varepsilon}{\Delta T} \frac{\partial T}{\partial y}\right) \frac{\partial T}{\partial y}= \\
\kappa_{\infty}\left(1+\frac{\varepsilon}{\Delta T}\left(T-T_{\infty}\right)+\frac{1}{\rho c_{p}} \frac{16 \sigma^{*} T_{\infty}^{3}}{3 K^{*}}\right) \frac{\partial^{2} T}{\partial y^{2}}+ \\
Q_{s}\left(T-T_{\infty}\right)+\frac{\mu}{\rho c}\left(\frac{\partial u}{\partial y}\right)^{2} .
\end{gathered}
$$

From Eq. (18) it is observed that the effect of variable thermal conductivity parameter $\varepsilon$ and thermal radiation parameter is to enhance the thermal diffusivity. The appropriate non-isothermal boundary conditions are; 


$$
\begin{aligned}
& \left.\begin{array}{l}
T=T_{w}\left[=T_{\infty}+A\left(\frac{x}{l}\right)^{2}\right] \quad \text { (PST Case) } \\
-k \frac{\partial T}{\partial y}=q_{w}=D\left(\frac{x}{l}\right)^{2} \quad \text { (PHF Case) }
\end{array}\right\} \quad \text { at } y=0 \\
& T \rightarrow T_{\infty} \quad \text { as } y \rightarrow \infty
\end{aligned}
$$

where $\mathrm{A}$ and $\mathrm{D}$ are constants. It is obvious now that,

$$
\Delta T=T_{w}-T_{\infty}= \begin{cases}A\left(\frac{x}{l}\right)^{2} & \text { PST Case } \\ \frac{D}{\kappa_{\infty}}\left(\frac{x}{l}\right)^{2} \sqrt{\frac{\gamma}{b}} & \text { PHF Case }\end{cases}
$$

We now use a scaled $\eta$-dependent temperature of the form,

$$
\theta(\eta)=\frac{T-T}{\Delta T}
$$

The advantage of Eq. (19) is that the temperaturedependent thermal conductivity turns out to be $x$ independent. Using Eq. (8) we reduce Eq. (18) to

$$
\left((1+\varepsilon \theta+N r) \theta^{\prime}\right)^{\prime}+\operatorname{Pr} f \theta^{\prime}-\operatorname{Pr}\left(2 f^{\prime}-\alpha\right) \theta+E c \operatorname{Pr} f^{\prime \prime 2}=0
$$

The parameters $\operatorname{Pr}, \alpha, E c$, and $N r$ are the Prandtl number, heat source/sink parameter, the Eckert number and the thermal radiation parameter respectively, and

$$
\begin{aligned}
& \text { are } \quad \text { by, } \quad \operatorname{Pr}=\frac{\mu C_{p}}{k_{\infty}}, \\
& \alpha=\frac{Q_{S}}{\rho c_{p} b}, \quad E c=\frac{b^{2} l^{2}}{A c_{p}}, \quad N r=\frac{16 \sigma^{*} T_{\infty}^{3}}{3 K^{*} k_{\infty}} .
\end{aligned}
$$

Using Eqs. (21), (20) one can reduce Eq. (19) to

$$
\left.\begin{array}{ll}
\theta(0)=1 & (\text { PST Case }) \\
\theta^{\prime}(0)=-1 & (\text { PHF Case })
\end{array}\right\}, \quad \theta(\infty)=0
$$

The local Nusselt number is given by

$$
N u_{x}=\frac{h(x)}{\mathrm{K}_{\infty}}
$$

where the heat transfer coefficient $h(x)$ is of the form

$$
h(x)=\frac{q_{w}(x)}{\Delta T}=-\frac{K_{\infty}}{\Delta T}\left(\frac{\partial T}{\partial y}\right)_{\text {at } y=0}=-K_{\infty}\left(\frac{\gamma}{b}\right)^{1 / 2} \theta^{\prime}(0)
$$

Substituting Eqs. (20), (21) and (25) into Eq. (24), we get

$$
N u_{x}=-\left(\frac{\gamma}{b}\right)^{1 / 2} \theta^{\prime}(0) \text {. }
$$

\section{Numerical Procedure}

The The transformed non-linear coupled ordinary differential Eqs. (9) and (22) with the boundary conditions (10) and (23) are solved numerically by a finite difference scheme known as Keller-Box method (Cebeci and Bradshaw 1984, Keller 1992).
The numerical solutions are obtained in four steps are as follows:

- reduce Eqs. (9) and (22) to a system of firstorder equations;

- $\quad$ write the difference equations using central differences;

- linearize the algebraic equations by Newton's method, and write them in matrix-vector form; and

- $\quad$ solve the linear system by the block tridiagonal elimination technique.

For the sake of brevity further details on the solution process are not presented here. It is also important to note that the computational time for each set of input parameter values should be short. Because physical domain in this problem is unbounded, whereas the computational domain has to be finite, we apply the far field boundary conditions for the similarity variable $\eta$ at finite value denoted by $\eta_{\max }$. We ran our bulk computations with the value $\eta_{\max }=10$, which is sufficient to achieve the far field boundary conditions asymptotically for all values of the parameters considered. For numerical calculations, a uniform step size of $\Delta \eta=0.01$ is found to be satisfactory and the solutions are obtained with an error tolerance of $10^{-6}$ in all the cases. In order to get a clear insight in to the physical problem, the numerical results for the horizontal velocity field $f^{\prime}(\eta)$ and temperature field $\theta(\eta)$, skin friction $f^{\prime \prime}(0)$ and wall-temperature gradient in PST Case and wall temperature in PHF case, are presented in Figs. 1-9. Furthermore, the salient features are discussed in section 5 .

\section{RESUlts AND DiscusSiON}

Figure 1 illustrates the effects of Maxwell parameter $\beta$ and the magnetic parameter $M n$ on the horizontal velocity profiles $f^{\prime}(\eta)$ with $\eta$. It is noticed from the figure that the horizontal velocity profiles $f^{\prime}(\eta)$ decrease with increasing values of $\beta$ and $M n$ in the boundary layer, but this effect is not very prominent near the wall. The effect of increasing value of $\beta$ is to reduce the horizontal velocity $f^{\prime}(\eta)$ and thereby reducing boundary layer thickness. That is, the thickness is much larger non-zero values of $\beta$, as clearly seen from Fig. 1. Further, from Fig. 1, can be seen that the horizontal velocity $f^{\prime}(\eta)$ decreases with an increase in the magnetic field parameters $M n$. This is due to the fact that, the introduction of transverse magnetic field (normal to the flow direction) has a tendency to create a drag, known as the Lorentz force which tends to resist the flow. This behavior is even true in the case of increasing values of the Maxwell parameter. 


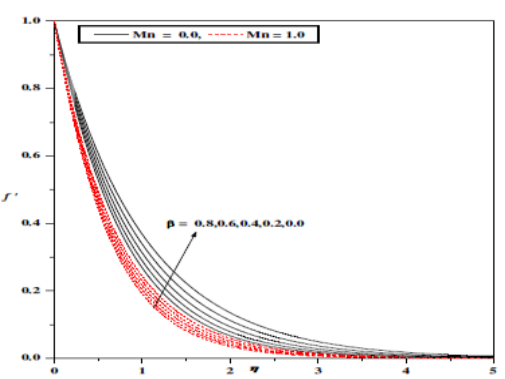

Fig. 1. Horizontal velocity profile $f^{\prime} V s \eta$ for different values of $\beta$ and $M n$

The effects of the Maxwell parameter $\beta$, the magnetic parameter $M n$, the thermal conductivity parameter $\varepsilon$, the heat source/sink parameter $\alpha$, the thermal radiation parameter $N r$, and the Eckert number $E c$ on temperature profile $\theta(\eta)$ with $\eta$ for non-isothermal boundary conditions (both PST and PHF cases) are shown graphically in Figs. 2-7. The general trend is that the temperature distribution $\theta(\eta)$ unity at the wall in PST case and is not unity at the wall in PHF case. However, the temperature distribution $\theta(\eta)$ for both PST and PHF cases decrease asymptotically to zero as the distance increases from the boundary. The effects of Maxwell parameter $\beta$ and the magnetic parameter $M n$ on temperature profiles $\theta(\eta)$ with $\eta$ in the boundary layer for both PST and PHF cases are shown respectively, in Figs. 2(a) and 2(b). The effect of increasing the values of $\beta$ leads to enhance the thermal boundary thickness. This is because of the fact that the thickening of the thermal boundary layer occurs due to an increase in the elasticity stress parameter.

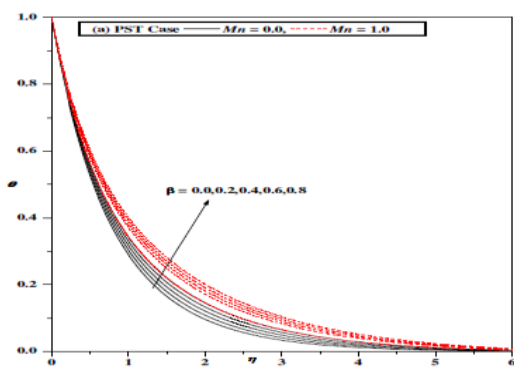

(a)

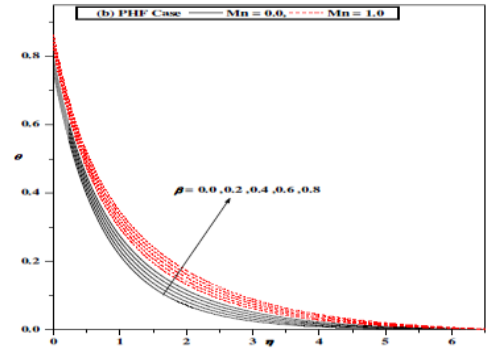

(b)

Fig. 2. (a) Temperature profile $\theta V s \eta$ for different values of $\beta$ and $M n$ when

$\operatorname{Pr}=1.0, \varepsilon=\alpha=E c=N r=0.0$,(b) Temperature profile $\theta V s \eta$ for different values of $\beta$ and $M n$ when $\operatorname{Pr}=1.0, \varepsilon=\alpha=E c=N r=0.0$
The behavior is even true for non-zero values of the magnetic parameter $M n$. As explained above, the introduction of a transverse magnetic field to an electrically conducting fluid gives rise to a resistive type of force known as Lorentz force. This force makes the fluid experience a resistance by increasing the friction between its layers and due to which there is increase in the temperature profile $\theta(\eta)$.

The effect of thermal conductivity parameter $\varepsilon$ on the temperature profile $\theta(\eta)$ with $\eta$ in the boundary layer for non-isothermal boundary conditions is shown graphically in Fig. 3. The profiles demonstrate quite clearly that an increase in the value of $\varepsilon$ results in an increase in the temperature profile $\theta(\eta)$ and hence the thermal boundary layer thickness increases as $\varepsilon$ increases. This is due to the fact that the assumption of temperature-dependent thermal conductivity causes a reduction in the magnitude of the transverse velocity by a quantity $\partial k(T) / \partial y$ as can be seen from heat transfer Eq. (18). This phenomenon holds for PHF case; however, thickness of the thermal boundary layer is smaller in comparison with PST case.

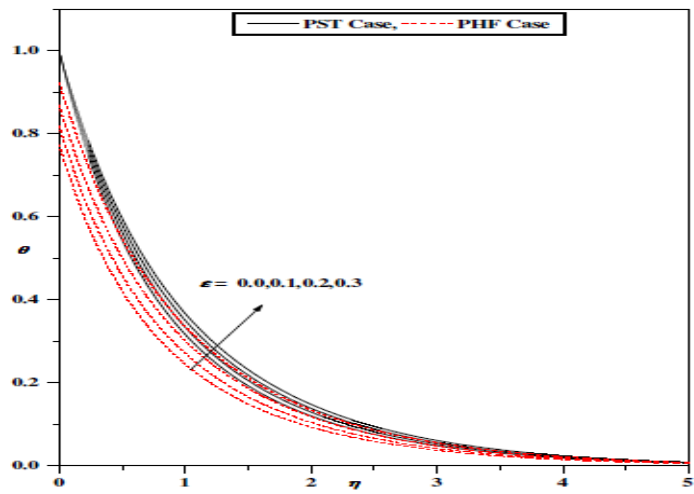

Fig. 3. Temperature profile $\theta V s \eta$ for different values of $\varepsilon$ with $\beta=0.4, M n=0.5, E c=N r=\alpha=0.0$, $\operatorname{Pr}=1.0$

In Fig. 4 the temperature distribution $\theta(\eta)$ for different values of the heat source/sink parameter $\alpha$ is shown. The dimensionless temperature attains unity at the wall for prescribed surface temperature and reduces to zero in the free stream for different values of heat source/sink parameter. However the temperature distribution for prescribed wall heat flux is different (less than unity) for different values of $\alpha$ at the surface and reduces to zero in the free stream. From this Figure we examine that the temperature profile is lower throughout the boundary layer for negative values of $\alpha$ (heat sink) and higher for positive values of $\alpha$ (heat source). Physically $\alpha>0$ implies $T_{w}>T_{\infty}$ i.e. there is a supply of heat to the flow region from the wall. Similarity $\alpha<0$ implies $T_{w}<T_{\infty}$ and there is a transfer of heat from the fluid to the wall. The effect of increasing value of heat source/ sink parameter $\alpha$ is to increase the temperature $\theta(\eta)$ in both PST and PHF cases. The graphs for the temperature distribution 
$\theta V s . \eta$ for different values of the Eckert number $E c$, the thermal radiation parameter $N r$, and the Prandtl number $P r$, for non-isothermal boundary conditions are plotted graphically in Figs. 5-7. We noticed from the curves that the effect of increasing values of $E c$ to enhance the temperature distribution $\theta(\eta)$. This is in conformity with the fact that the energy is stored in the fluid region, as a consequence of dissipation due to viscosity and elastic deformation, as shown in Fig. 5.

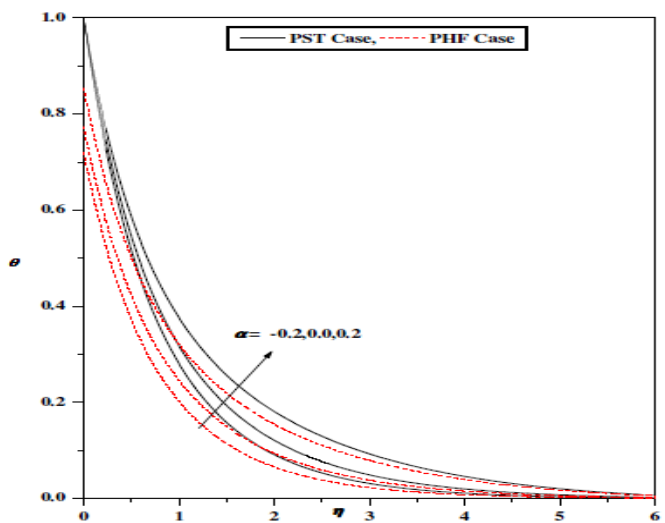

Fig. 4. Temperature profile $\theta$ Vs $\eta$ for different values of $\alpha$ with $\beta=0.4, M n=0.5, E c=N r=0.0, \varepsilon=0.1$, $\operatorname{Pr}=1.0$.

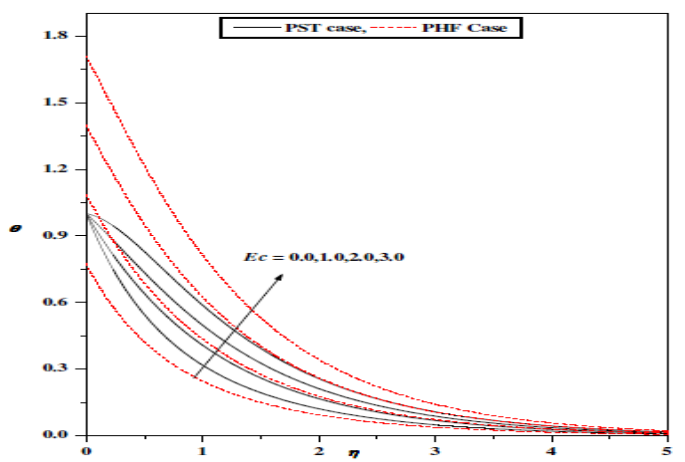

Fig. 5. Temperature profile $\theta V s \eta$ for different values of $E c$ with $\beta=0.4, M n=0.5, \alpha=N r=0.0, \varepsilon=0.1$, $\operatorname{Pr}=1.0$.

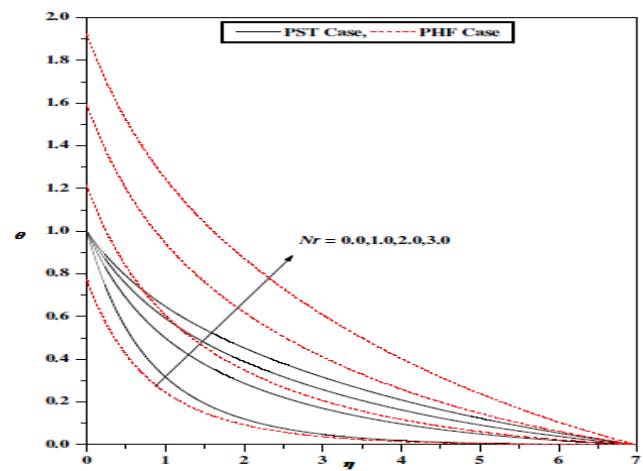

Fig. 6. Temperature profile $\theta$ Vs $\eta$ for different values of $N r$ with $\beta=0.4, M n=0.5, \alpha=E c=0.0, \varepsilon=0.1$,

$$
\operatorname{Pr}=1.0 \text {. }
$$

The effect of increasing values of $N r$ is to increase the temperature profile $\theta(\eta)$ and hence increases the thermal boundary layer thickness; depicted in Fig. 6 . This result qualitatively agrees well with the fact that the effect of thermal radiation is to enhance the rate of transport to the fluid, thereby increasing the temperature of the fluid. Further it is observed from the Figs. 5 and 6 that, the region of the thermal boundary layer is more pronounced for PHF case in comparison with PST case for non-zero values of the Eckert number and the thermal radiation parameter. The profiles in Fig. 8 exhibit the role of Prandtl number on temperature profile $\theta(\eta)$. The effect of increasing values of $\mathrm{Pr}$ results in decrease of the temperature distribution and hence thermal boundary layer thickness decreases as $\mathrm{Pr}$ increases. This phenomenon is true in both PST and PHF cases. However, from the Fig. 7, it is noticeable that the thickness of the thermal boundary layer is larger in PST case as compared to the PHF case.

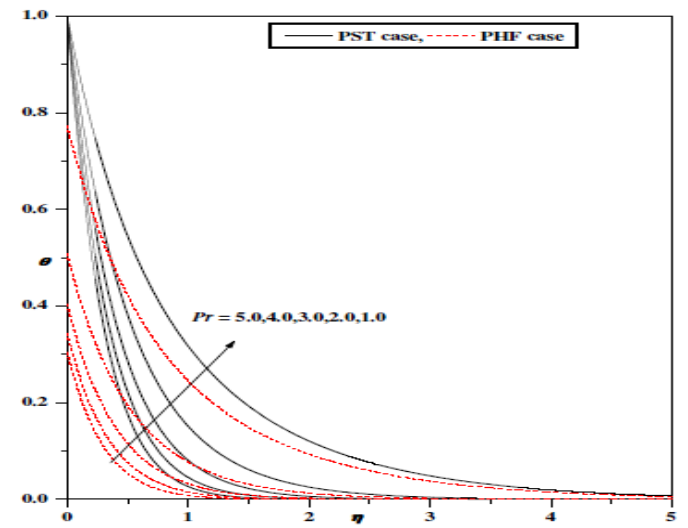

Fig. 7. Temperature profile $\theta V s \eta$ for different values of $\operatorname{Pr}$ with $\beta=0.4, M n=0.5, \alpha=E c=0.0, \varepsilon=0.1$.

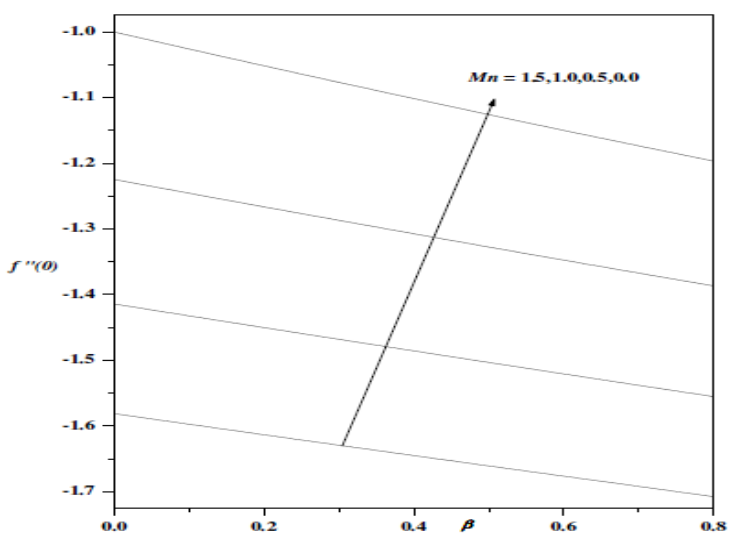

Fig. 8. Values of skin friction $f^{\prime \prime}(0) V s \beta$ for different values of $M n$.

Figurs. 8 and 9 display the variation of skin friction $f^{\prime \prime}(0)$, wall temperature gradient $\theta^{\prime}(0)$ (PST Case) and wall temperature $\theta(0)$ (PHF case) vs. the Maxwell parameter for zero and non-zero values of the magnetic parameter. It can be noted that the skin friction decreases with an increase in the Maxwell parameter as 
well as with the magnetic parameter; whereas quite opposite holds for non-isothermal temperature boundary conditions.

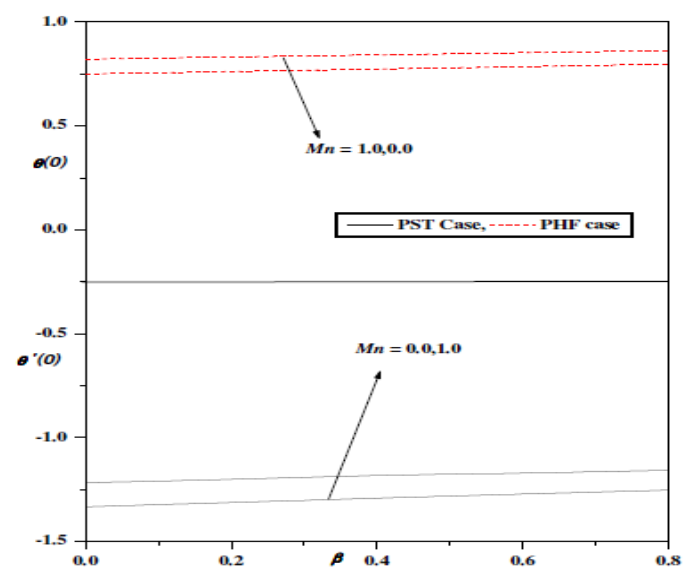

Fig. 9. Values of skin friction $\theta^{\prime}(0) V s \beta$ (PST case), $\theta$ (0) $V s \beta$ (PHF case) for different values of $M n$ with

$$
E c=N r=\alpha=0.0, \operatorname{Pr}=1.0 \text {. }
$$

\section{REFERENCES}

Agassant, J. F., P. Avens, J. Sergent and P. J. Carreau, (1991). Polymer Processing: Principles and Modeling. Hanser Publishers, Munich.

Andersson, H.I. (1992). MHD flow of a visco-elastic fluid past a stretching surface. Acta Mechanica, $95,227-230$.

Aliakbar, V., A. Alizadeh-Pahlavan and K. Sadeghy (2009). The influence of thermal radiation on MHD flow of Maxwellian fluids above stretching sheets. Communications in Nonlinear Science and Numerical Simulation 14(3), 779794.

Bhatnagar, R. K., G. Gupta and K. R. Rajagopal, (1995). Flow of an Oldroyd-B fluid due to a stretching sheet in the presence of a free stream velocity. International Journal of Non-Linear Mechanics 30(3), 391-405.

Brewster, M.Q. (1992). Thermal radiative transfer properties. John Wiley and Sons, New York.

Bird, R.B., Armstrong, R.C., and Hassager, O. (1987). Dynamics of polymeric liquids. John Wiley and Sons, New York.

Cebeci, T. and P. Bradshaw (1984). Physical and computational aspects of convective heat transfer. Springer-Verlag, New York.

Chaim, T. C. (1998). Heat transfer in a fluid with variable thermal conductivity over a linearly stretching sheet, Acta Mechanica. 129(1-2), 6372 .
Chen, C. K. and M. I. Char, (1988). Heat transfer of a continuous stretching surface with suction or blowing. Journal of Mathematical Analysis and Applications, 135(2), 568-580.

Cortell, R. (2005). Flow and heat transfer of a fluid through a porous medium over a stretching surface with internal heat generation/absorption and suction/blowing. Fluid Dynamics Research, 27(5), 231-245.

Cortell, R. (2006). Flow and heat transfer of an electrically conducting fluid of second grade over a stretching sheet subject to suction and to a transverse magnetic field. International Journal of Heat and Mass Transfer, 49(11-12), 1851-1856.

Crane, L. J. (1970). Flow past a stretching plate, Zeitschrift für angewandte Mathematik und Physik ZAMP, 21(4), 645-647.

Grag, V. K. and K. R. Rajagopal (1991). Flow of a non-Newtonian fluid past a wedge. Acta Mechanica, 88(1-2), 113-123.

Govindarajulu, T. C. J. and Thangaraj (1987). A Note on Thermal Boundary Layer in Liquid Metals with Variable Thermal Conductivity. Journal of Applied Mathematics and Mechanics / Zeitschrift für Angewandte Mathematik und Mechanik, ZAMM, 67(12), 657-660.

Grubka, L. J. and K. M. Bobba (1985). Heat transfer characteristics of a continuous stretching surface with variable temperature. ASME J. Heat Trans. 107, 248-250.

Gupta, P. S. and A. S. Gupta (1977). Heat and mass transfer on a stretching sheet with suction or blowing. The Canadian Journal of Chemical Engineering. 55(6), 744-747.

Gupta, A. S. and A. S. Wineman (1980). On a boundary layer theory for non-Newtonian fluids. Letters in Applied Engineering Science, 18, 875883.

Hayat, T., Z. Abbas and M. Sajid (2006). Series solution for the upper-Convected Maxwell fluid over a porous stretching plate. Physics Letters A, 358(5-6), 396-403.

Keller, H. B. (1992). Numerical Methods for Two-point Boundary Value Problems, Dover Publ., New York.

Liu, I. C. (2005). A note on heat and mass transfer for hydromagnetic flow over a stretching sheet. International Communications in Heat and Mass Transfer. 32(8), 1075-1084.

Rajagopal, K. R. and A. S. Gupta, (1985). An exact solution for the flow of a non-Newtonian fluid past an infinite porous plate. Meccanica, 19(2), 158-160. 
K. V. Prasad et al. / JAFM, Vol. 6, No. 2, pp. 249-256, 2013.

Rajagopal, K. R., T. Y. Na and A. S. Gupta (1984). Flow of a viscoelastic fluid over a stretching sheet. Rheologica Acta,. 23(2), 213-215.

Raptis, A. and C. Perdikis (1998). Viscoelastic flow by the presence of radiation. Journal of Applied Mathematics and Mechanics / Zeitschrift für Angewandte Mathematik und Mechanik ZAMM, 78(4), 277-279.

Raptis, A. (1999). Radiation and visco elastic flow, International Communications in Heat and Mass Transfer, 26(6), 889-892.

Renardy, M. (1997). High weissenberg number boundary layers for upper convected Maxwell Fluid. Journal of Non-Newtonian Fluid Mechanics, 68(1), 125-132.

Sadeghy, K., A .H. Najafi, and M. Saffaripour (2005). Sakiadis flow of an upper-convected Maxwell fluid. International Journal of Non-Linear Mechanics, 40(9), 1220-1228.

Sakiadis, B. C. (1961). Boundary layer behavior on continuous solid surfaces. AIChE Journal. 7(1), 26-28.
Savvas, T. A., N. C. Markatos and C. D. Papaspyrides, (1994). On the flow of non-Newtonian polymer solutions. Applied Mathematical Modelling,18(1), 14-21.

Siddappa, B., and M. Subhas Abel (1985). NonNewtonian flow past a stretching plate. Zeitschrift für angewandte Mathematik und Physik ZAMP, 36(6), 890-892.

Subhas Abel, M., E. Sanjayanand and M. N. Mahantesh (2008). Viscoelastic MHD flow and heat transfer over a stretching sheet with viscous and ohmic dissipations. Communications in Nonlinear Science and Numerical Simulation, 13(9), 1808-1821.

Subhas Abel, M. and N. Mahesha (2008). Heat transfer in MHD viscoelastic fluid flow over a stretching sheet with variable thermal conductivity, nonuniform heat source and Radiation. Applied Mathematical Modelling, 32(10), 1965-1983.

Vleggaar, J. (1977). Laminar boundary layer behaviour on continuous accelerating surfaces. Chemical Engineering Science. 32(12), 1517-1525. 